\title{
Additivity of Quadratic Maps
}

\author{
By \\ Yaakov FRIEDMAN* and Josuke HAKEDA**
}

\begin{abstract}
The set of observables of a Quantum Mechanical System need only be closed under a "quadratic" product. It is shown that an additive structure of this set (whose existence is less natural) is uniquely determined by this multiplicative structure.
\end{abstract}

\section{$\S 0$. Introduction}

Jordan algebras were introduced by Jordan, von Neumann, and Wigner [13] as a model for the observables in a quantum mechanical system. Emch [4] provides further justification for this algebraic model. Although there is no doubt about the naturalness of the multiplicative structure, the physical meaning of the additive structure is unclear. Yet an additive structure is necessary to perform calculations, and thus we are led to consider representations of the multiplicative structure in linear spaces. A basic question arises: must the induced map between two such representations be linear?

An examination of the formulations of measurable quantities in quantum mechanics reveals that all such quantities can be expressed in terms of a quadratic operation. (cf. [6]). In associative algebras this product has the form $x y x$. Abstractly, we shall speak of a quadratic product of $x$ and $y$ and a set which is closed under the quadratic product will be called a quadratic semigroup. Evidently, the observables of a quantum mechanical system need be closed only under the quadratic maps i.e., they form a quadratic semigroup.

We consider representations of quadratic semigroups in linear spaces. In this context the quadratic product can always be linearized to determine a ternary algebraic structure on the linear space. Among all Banach spaces, the $J B^{*}$ triples (defined below) are the only ternary algebras known at this time whose multiplicative structure is a quadratic semigroup. We formulate the question of the first paragraph in this context: must a bijection between two $J B^{*}$-triples

Communicated by H. Araki, December 22, 1987.

* Department of Mathematics, Jerusalem College of Technology, P. O. Box 16031, Jerusalem 91-160, Israel.

** Department of Basic Technology, Yamagata University, Yonezawa 992, Japan.

This work is partially supported by NSF. 
which preserves the quadratic product be linear? We give a positive answer to this question in this article.

The quadratic map is related to the squaring map, which was used by Segal in [19] to give an Axiomatics of Quantum Mechanics. We want to mention that the model based on squaring maps leads automatically to ordered spaces, while the quadratic maps could be defined also in non-ordered spaces.

In section 1 we will define $J B^{*}$-triples and recall the background needed to read this article. In section 2 the main result will be proven. Namely, we will show that any mapping between $J B W^{*}$-triples (with no abelian component) which preserves quadratic products, is the sum of a linear isomorphism and a conjugate linear isomorphism. In the final section our main result will be used to prove uniqueness of a representation of an involutive semigroup.

This work was done while the second-named author was visiting the Department of Mathematics, University of California, Irvine. He would like to express his sincere thanks to Professor Bernard Russo for hospitality and for valuable discussions concerning $J B^{*}$-triples. Both authors wish to thank him for calling our attention to an error in the first draft and to thank Professor T. Barton and a referee for helpful discussions.

\section{$\S 1$. Preliminaries on $J B^{*}$-triples}

$J B^{*}$-triples are the algebraic structure associated with bounded symmetric domains in Banach spaces [15], [20]. They contain the class of $C^{*}$-algebras and $J B^{*}$-algebras and could be considered as their generalization. The usual definition of $J B^{*}$-triple is as follows:

Definition 1. A Banach space $U$ over $\mathbf{C}$ is said to be a $\boldsymbol{J B}^{*}$-triple if it is equipped with a triple product $(a, b, c) \rightarrow\{a b c\}$ mapping $U \times U \times U$ to $U$ such that:

(i) $\{a b c\}$ is linear in $a$ and $c$ and conjugate linear in $b$;

(ii) $\{a b c\}$ is symmetric in the outer variables i.e., $\{a b c\}=\{c b a\}$;

(iii) for any $x \in U$, the operator $\delta(x)$ from $U$ to $U$ defined by:

$$
\delta(x) y=\{x x y\}, \quad y \in U
$$

is hermitian (i.e., $\exp (i t \delta)$ is an isometry for all real $t$ ) with nonnegative spectrum;

(iv) the product satisfies the following identity, called the "main identity";

$$
\delta(x)\{a b c\}=\{(\delta(x) a) b c\}-\{a(\delta(x) b) c\}+\{a b(\delta(x) c)\} ;
$$

(v) the following norm condition holds:

$$
\|\delta(x)\|=\|x\|^{2} .
$$


If $U$ is a $C^{*}$-algebra, then $U$ becomes a $J B^{*}$-triple with the product

$$
\{a b c\}=\frac{a b^{*} c+c b^{*} a}{2} \text {. }
$$

Moreover, any closed subspace of $B(H)$ (the space of bounded operators on a Hilbert space $H$ ) closed under this product is a $J B^{*}$-triple. If $U$ is a $J B^{*}$ algebra, it becomes a $J B^{*}$-triple with the product

$$
\{a b c\}=\left(a \circ b^{*}\right) \circ c+a \circ\left(b^{*} \circ c\right)-(a \circ c) \circ b^{*} .
$$

See $[20]$ for the proof.

The triple product of a $J B^{*}$-triple is determined by the geometry of the unit ball in these spaces and any linear isometry preserves the triple product structure [15], [20].

To describe the multiplicative structure of a $J B^{*}$-triple, introduce a conjugate linear operator $Q(x)$ for any $x \in U$, defined by :

$$
Q(x) y=\{x y x\}, \quad y \in U .
$$

The following definition describes the "multiplicative semigroup" structure of a $J B^{*}$-triple:

Definition 2. We shall say that an arbitrary set $G$ is a quadratic semigroup if there is a mapping $(a, b) \rightarrow Q(a) b$ of $G \times G \rightarrow G$ such that:

$$
Q(Q(x) y) z=Q(x) Q(y) Q(x) z
$$

for any $x, y, z \in G$.

It is known [17], [20] that in a Jordan triple system (in particular a $J B^{*}$. triple), the main identity (1.2) implies (1.6). Since the main identity also involves addition, it cannot be used to describe the multiplicative structure of a $J B^{*}$. triple.

The basic building blocks for $J B^{*}$-triples are the tripotents, defined as elements of $U$ satisfying:

$$
u=Q(u) u \text {. }
$$

The set of tripotents is a partially ordered set with order

$$
v \leqq u \quad \text { if and only if } v=Q(v) u \text {. }
$$

Two tripotents $u$ and $v$ are said to be orthogonal if $u+v$ and $u-v$ are tripotents. It is known [16] that the condition $u$ is orthogonal to $v$ (denoted by $u \perp v)$ is equivalent to

$$
\{u u v\}=0, \quad\{v v u\}=0 \quad \text { or } \quad\{u v U\}=\{v u U\}=0 .
$$


For such $u$ and $v$ we also have

$$
u+v \geqq u \text { and } u+v \geqq v .
$$

A tripotent $u$ is said to be minimal if $0 \neq v \leqq u$ (and $v$ a tripotent) implies $u=v$. If $u$ is a minimal tripotent then (cf. [16]) for any $x \in U$ there is $\alpha \in \mathbf{C}$ such that

$$
\{u x u\}=\alpha u \text {. }
$$

An important tool for studying $J B^{*}$-triples are Peirce projections and Peirce decompositions with respect to any tripotent $u$. It is easy to verify from the definition that for a tripotent $u$, the spectrum of the operator $\delta(u)$, defined by (1.1), is contained in the set $\{0,1 / 2,1\}$ (see [20] for details). Thus $U$ can be decomposed into eigenspaces of this operator:

$$
U=U_{2}(u)+U_{1}(u)+U_{0}(u)
$$

where $x \in U_{k}(u)$ if and only if

$$
\delta(u) x=\{u u x\}=\frac{k}{2} x
$$

for $k=0,1,2$.

From this and (1.2) follow the Peirce rules:

$$
\left\{U_{k}(u) U_{\jmath}(u) U_{l}(u)\right\} \subset U_{k-\jmath+l}(u)
$$

for any $k, j, l \in\{0,1,2\}$ and $\left\{U_{k}(u) U_{j}(u) U_{l}(u)\right\}=\{0\}$ if $k-j+l \notin\{0,1,2\}$.

Moreover, it can be shown [16] that

$$
\left\{U_{k} U_{\jmath} U\right\}=\left\{U U_{\jmath} U_{k}\right\}=\{0\}
$$

when $\{k, j\}=\{0,2\}$.

The subspace $U_{2}(u)$ has a binary structure defined by

$$
x \circ y=\{x u y\} \quad \text { for } x, y \in U_{2}(u) .
$$

With this product $U_{2}(u)$ becomes a $J B^{*}$-algebra with identity $u$ and involution

$$
x^{\#}=\{u x u\}=Q(u) x .
$$

For more about the structure of this $J B^{*}$-algebra, see [20: Section 19]. Note that for arbitrary $x \in U$, from (1.16) it follows that

$$
\{u x u\} \in U_{2}(u)
$$

and any element of $U_{2}(u)$ is of this form.

For any tripotent $u \in U$ and $k=0,1,2$ there are contractive projections $P_{k}(u)$ from $U$ onto $U_{k}(u)$. These projections are: 


$$
\begin{aligned}
& P_{2}(u)=Q(u)^{2} \\
& P_{1}(u)=1-2 \delta(u)+Q(u)^{2} \\
& P_{0}(u)=2\left(\delta(u)-Q(u)^{2}\right) .
\end{aligned}
$$

Sometimes we need to decompose $U$ relative to two tripotents $u$ and $v$. If these decompositions commute i.e., $\left[P_{\jmath}(u), P_{k}(v)\right]=0$ for any $j, k \in\{0,1,2\}$, we will say that $u$ and $v$ are compatible. It was shown [18] that $u$ and $v$ are compatible if and only if $\{u u v\} \in U_{2}(v)$. For instance if $v \in U_{k}(u)$ for some $k$, then $u$ and $v$ are compatible. Also if $u$ and $v$ are compatible and $P_{2}(u) v=0$ then $P_{1}(u) v$ and $P_{0}(u) v$ are orthogonal tripotents. See for details, Corollary 1.8 of $[18]$.

In addition to the relation of orthogonality defined above, we will need two other relations between tripotents. We will say that $u$ is collinear to $v$ if:

$$
u \in U_{1}(v), \quad v \in U_{1}(u)
$$

and $w$ is said to govern $u$ if

$$
w \in U_{1}(u) \text { and } u \in U_{2}(w) .
$$

The following simple lemmas about these relations will be used later.

Lemma 1.1. Let $w, u$ be tripotents and suppose that $w$ governs $u$. Then $v=$ $Q(w) u$ is a tripotent orthogonal to $u$. Moreover $p=1 / 2(u+v+w)$ and $q=$ $1 / 2(u+v-w)$ are a pair of orthogonal tripotents.

Proof. By [3, Proposition 1.6] $v$ is a tripotent orthogonal to $u, w$ governs $v, u=Q(w) v, Q(u+v) w=w$ and $Q(w)(u+v)=u+v$. Thus $Q(u+v+w)(u+v+w)$ $=Q(u+v)(u+v+w)+Q(w)(u+v+w)+2\{(u+v)(u+v+w) w\}=2(u+v+w)+$ $2[\{(u+v)(u+v) w\}+\{(u+v) w w\}]=4(u+v+w)$ implying $p$ is a tripotent. Similarly one checks that $q$ is a tripotent. Since $p+q=u+v$ and $p-q=w$ are tripotents, $p$ is orthogonal to $q$.

Lemma 1.2. Let $u, v$ and $w$ be tripotents, $u \in U_{2}(v)$ and suppose that $w$ governs $v$. Then there is a tripotent $w_{1}$ governing $u$.

Proof. Let $v^{\prime}=Q(w) v$ and $u^{\prime}=Q(v) u$. Then $v=Q(w) v^{\prime}$ and $u=Q\left(v^{\prime}\right) u^{\prime}=$ $Q\left(Q(w) v^{\prime}\right) u^{\prime}=Q(w) Q\left(v^{\prime}\right) Q(w) u^{\prime} \in U_{2}(w)$. Thus by [18], $u$ and $w$ are compatible. Moreover, $u \in U_{2}(v)$ and $w \in U_{1}(v)$ implies $P_{2}(u) w=P_{2}(u) P_{2}(v) w=0$ and thus $w=$ $P_{1}(u) w+P_{0}(u) w:=w_{1}+w_{0}$ where $w_{1}$ and $w_{0}$ are orthogonal tripotents [18: Rk. 1.9]. Since $w_{0} \perp u$, we have $u=P_{2}(w) u=Q\left(w_{1}+w_{0}\right)^{2} u=Q\left(w_{1}+w_{0}\right)\left[Q\left(w_{1}\right) u+\right.$ $\left.2\left\{w_{1} u w_{0}\right\}+Q\left(w_{0}\right) u\right]=Q\left(w_{1}+u_{0}\right) Q\left(w_{1}\right) u=Q\left(w_{1}\right)^{2} u=P_{2}\left(w_{1}\right) u$, that is, $w_{1}=P_{1}(u) w \in$ $U_{1}(u)$ and $u=P_{2}\left(w_{1}\right) u \in U_{2}\left(w_{1}\right)$, i. e. $w_{1}$ governs $u$. 
$J B^{*}$-triples in general may not have tripotents, so we will consider only $J B W^{*}$-triples defined as follows: $U$ is said to be a $J B W^{*}$-triple if $U$ is a $J B^{*}$ triple and is the dual of a Banach space $U_{*}$, called the predual of $U$.

It is known that the second dual of a $J B^{*}$-triple is a $J B^{*}$-triple [2], and that the product in a $J B W^{*}$-triple is separately $w^{*}$-continuous [1]. For any element $x$ of a $J B W^{*}$-triple $U$ there is a polar decomposition assigning to $x$ a unique tripotent $u$ (called the support tripotent of $x$ ) such that $u$ belongs to the smallest $J B W^{*}$-subtriple containing $x$, and $x$ is strictly positive in the $J B W^{*}$ algebra $U_{2}(u)$. Moreover if $x \in U_{k}(v)$ then also $u \in U_{k}(v)$, see [12:3.14]. Two elements $x, y$ in $U$ are said to be orthogonal if there is a tripotent $u$ such that $x \in U_{2}(u)$ and $y \in U_{0}(u)$. For any element $f \in U_{*}$ there is a unique tripotent $u=u(f)$ in $U$ such that:

$$
f=f \circ P_{2}(u)=f \circ Q(u)^{2}
$$

and $f$ is a positive faithful functional on $U_{2}(u)$ [5: Prop. 2].

A subspace $W$ of $U$ is said to be an ideal if $\{a b c\} \in W$ whenever one of the elements $a, b$ or $c$ is in $W$. We say that $W$ is a summand if there is a subspace $\widetilde{W}$ of $U$ such that any $x \in W$ is orthogonal to any $y \in \widetilde{W}$ and $U=W \oplus \widetilde{W}$. It is known [10], [11] that the $w *$-closed ideals in a $J B W^{*}$-triple $U$ coincide with the summands in $U$.

A $J B^{*}$-triple $W$ is said to be abelian if $\{a b\{c d e\}\}=\{\{a b c\} d e\}=\{a\{d c b\} e\}$ holds for all $a, b, c, d, e$ in $W$. For a $J B W^{*}$-triple this is equivalent to $W_{1}(u)$ $=0$ for all tripotents $u \in W$.

A tripotent $v$ in a $J B W^{*}$-triple $U$ is said to be an abelian tripotent if $U_{2}(v)$ is an abelian $J B W^{*}$-triple and $v$ is said to be a complete tripotent if $U_{0}(v)=0$. It is known that the extreme points of the Ball $U$ are the complete tripotents.

A $J B W^{*}$-triple is said to be of type $I$ if it is generated as a weak*-closed ideal by an abelian tripotent. It is known [10], [11] that any $J B W^{*}$-triple $U$ can be decomposed into a direct sum of $U_{I}$ and $U_{N I}$ where $U_{I}$ is of type $I$ and $U_{N I}$ does not contain abelian tripotents. A $J B W^{*}$-triple $W$ is said to be of type $I_{1}$ if $W$ contains a complete abelian tripotent. It is also shown in [10], [11] that $U_{I}$ can be decomposed into a direct sum $W \oplus \widetilde{W}$ where $W$ is of type $I_{1}$ and $\widetilde{W}$ does not contain type $I_{1}$ summands.

A type $I_{1} J B W^{*}$-triple $W$ can be decomposed further into a direct sum $W=$ $\oplus W_{n}$ for some set of cardinal numbers $n$ such that $W_{n}$ is of type $I_{1, n}$, defined as $J B W^{*}$-triples containing a colinear family $\left\{v_{\jmath}\right\}_{\jmath \in J}$ of $n$ complete abelian tripotents such that $W_{n}=\overline{\operatorname{span}}\left\{W_{2}\left(v_{j}\right): j \in J\right\}$, or equivalently $\cap\left\{W_{1}\left(v_{j}\right): j \in J\right\}$ $=\{0\}$.

A family $\left\{f_{i}\right\}_{i \in I}$ in $U_{*}$ is said to be complete if $f_{\imath}(s)=0$ for some $s \in U$ and all $i \in I$ implies $s=0$.

Definition 3. We will say that $f \in U_{*}$ has property $G$ (governing) if there 
is a tripotent $w$ in $U$ governing $v(f)$ (the support tripotent of $f$ ).

The following technical proposition plays an important role in the proof of the main result.

Proposition 1.3. Let $U$ be a $J B W^{*}$-triple with no type $I_{1}$ direct summand. Then there is a complete family $\left\{f_{\imath}\right\}_{i \in I}$ in $U_{*}$ such that for each $i \in I, f_{i}$ has property $G$.

Proof. Let $U=U_{I} \oplus U_{N I}$ where $U_{I}$ is of type $I$ and $U_{N I}$ has no type $I$ summands and let $\left\{f_{i}\right\}_{i \in I}$ consist of all points of Ball $\left(U_{I}\right)^{*}$ with an abelian support $v\left(f_{i}\right)$ and all $\left.\left.f \in\right) U_{N I}\right)^{*}$ satisfying property $G$.

By $[11: 5.9]$, for any given abelian tripotent $v$ in $U_{I}$, there is a complete tripotent $w$ in $U_{I}$ such that $v$ is an abelian $w$-projection in a type $I J B W^{*}$ algebra $U_{2}(w)$. By our assumption on $U_{I}, A=\left\{x \in U_{2}(w): x^{\#}=x\right\}$ is a $J B W^{*}$ algebra of type $I$ with no type $I_{1}$ summand. So, by [9: Lemma 5.3.2], there are a $w$-projection $u$ and a symmetry $s$ in $A$ such that $Q(s) v=u$ and $Q(s) u=v$. (Note that $u$ and $s$ are tripotents in $U)$. Since $Q(s)(u+v)=u+v, u+v$ and $s$ operator commute and so it follows that $Q(u+v) s$ is a $(u+v)$-self-adjoint symmetry in $U_{2}(u+v)(Q(u+v) s$ is a tripotent in $U)$. From (1.14) follows that also $\tilde{s}=P_{1}(u) P_{1}(v) Q(u+v) s$ satisfies $Q(\tilde{s}) v=u$ and $Q(\tilde{s}) u=v$. Moreover, since $Q(\tilde{s})(u+v)$ $=u+v, \tilde{s}$ is a self-adjoint symmetry in $U_{2}(u+v)$ and hence a tripotent in $U$ which is governing $v$. Thus any $f_{i}$ with abelian support $v\left(f_{\imath}\right)$ satisfies property $G$.

To show that such $f_{i}$ form a complete family in $\left(U_{I}\right)^{*}$ it is enough to show that the abeliam tripotents are $w^{*}$-dense in $U_{I}$. Indeed, if $g$ in $\left(U_{I}\right)^{*}$ vanishes on all abelian tripotents and is not zero, denote by $u$ its support tripotent. By [11] $U_{2}(u)$ is a type $I J B W^{*}$-algebra and therefore there is an abelian projection $v$ in this algebra which is an abelian tripotent. Thus $g(v)=0$ contradicts the faithfulness of $g$ on $U_{2}(u)$.

If $g \in\left(U_{N I}\right)^{*}$ and $u=u(g)$, then the $J B W^{*}$-algebra $U_{2}(u)$ has ro type $I$ direct summand. Thus by [9: Proposition 5.2.15] there are tripotents $u_{i}$ and $u_{i j}$ for $1 \leqq i, j \leqq 4, i \neq j$ such that $u_{i j}=u_{j i}, u_{i}$ orthogonal to $u_{j}$ if $i \neq j, u=u_{1}+u_{2}+u_{3}+u_{4}$ and $u_{i j}$ governs $u_{i}$. It is easy to verify that $u_{i k}+u_{j l}$ is a tripotent governing $u_{i}+u_{j}$ for $\{i, j, k, l\}=\{1,2,3,4\}$, and $\{i, k\} \cap\{j, l\}=\varnothing$. Thus each element of $\left\{u_{i}+u_{j}\right\}$ has a governing tripotent. Define $g_{i}=g \circ P_{2}\left(u_{i}\right)$ and $g_{i j}=g \circ P_{1}\left(u_{i}\right) P_{1}\left(u_{j}\right)$. Then $g=g \circ P_{2}(u)=\sum_{i} g_{i}+\sum_{i<j} g_{i j}$. Since $u\left(g_{i}\right) \in U_{2}\left(u_{\imath}\right)$ and $u\left(g_{i j}\right) \in U_{2}\left(u_{i}+u_{j}\right)$, from Lemma 1.2 it follows that $g_{i}$ and $g_{i}$ satisfy property $G$. Thus $g$ is in the span of $\left\{f_{i}\right\}_{i \in I}$. 


\section{§2. Main Result}

Definition 2.1. A map $\phi$ from a $J B^{*}$-triple $U$ to another complex $J B^{*}$ triple $V$ is called a quadratic map from $U$ to $V$ if $\phi$ satisfies

$$
\phi(Q(a) b)=\phi(\{a b a\})=\{\phi(a) \phi(b) \phi(a)\}=Q(\phi(a)) \phi(b) .
$$

Remark 2.2. Any quadratic map $\phi$ maps tripotents into tripotents. Moreover, since the order $e \leqq f$ on the set of tripotents is defined by $e=Q(e) f, \phi$ preserves the order of tripotents. If $\phi$ is bijective, then $\phi(0)=0$.

Lemma 2.3. Let $U$ and $V$ be $J B^{*}$-triples. Let $\phi$ be a bijective quadratic map from $U$ to $V$. Then $u$ and $v$ are orthogonal tripotents in $U$ if and only if $\phi(u)$ and $\phi(v)$ are orthogonal tripotents in $V$. Moreover,

$$
\phi(u+v)=\phi(u)+\phi(v) .
$$

Proof. Since $v \leqq u+v$, we have $\phi(v) \leqq \phi(u+v)$. Then there exists a tripotent $\tilde{w}$ in $V$ such that $\phi(u+v)=\phi(v)+\tilde{w}$ and $\tilde{w}$ is orthogonal to $\phi(v)$ (see [5: Cor. 1.7]). Hence,

$$
\begin{aligned}
\phi(u) & =Q(\phi(u)) \phi(u+v)=Q(\phi(u))(\phi(v)+\tilde{w}) \\
& =Q(\phi(u)) \phi(v)+Q(\phi(u)) \tilde{w} \\
& =\phi(Q(u) v)+Q(\phi(u)) \tilde{w} .
\end{aligned}
$$

Since $u$ and $v$ are orthogonal tripotents, we have $\phi(u)=Q(\phi(u)) \tilde{w}$, that is, $\phi(u) \leqq \tilde{w}$. Since $\tilde{w}$ is orthogonal to $\phi(v), \phi(u)$ is also orthogonal to $\phi(v)$.

Let $w:=\phi^{-1}(\tilde{w})$. Since $\phi(w) \leqq \phi(u+v)$, we have $\phi(w)=Q(\phi(u+v)) \phi(w)$. Moreover, since $\phi^{-1}$ is also a bijective quadratic map, it maps orthogonal tripotents $\phi(v)$ and $\phi(w)$ into orthogonal tripotents $v$ and $w$. Thus

$$
\begin{aligned}
\tilde{w}=\phi(w) & =Q(\phi(u+v)) \phi(w) \\
& =\phi(Q(u+v) w) \\
& =\phi(Q(u) w+2\{u w v\}+Q(v) w) \\
& =\phi(Q(u) w)=Q(\phi(u)) \phi(w)=\phi(u)
\end{aligned}
$$

and $\phi(u+v)=\phi(v)+\tilde{w}=\phi(u)+\phi(v)$.

Corollary 2.4. Let $U$ and $V$ be $J B W^{*}$-triples. Let $\phi$ be a bijective quadratic map from $U$ to $V$, then any Peirce subspace of a tripotent $u$ is mapped by $\phi$ onto the corresponding Peirce subspace of $\phi(u)$, i.e.,

$$
\phi \circ P_{k}(u)=P_{k}(\phi(u)) \circ \phi \circ P_{k}(u) \quad \text { for } k=2,1,0 .
$$

Proof. Let $u$ be an arbitrary tripotent in $U$. Then 


$$
\begin{gathered}
P_{2}(u) U=\left\{x \in U: Q(u)^{2} x=x\right\}, \\
P_{1}(u) U=\{x \in U: Q(u) x=0 \text { and } Q(v) x=0 \text { for all tripotents } v \\
\text { orthogonal to } u\}, \text { and } \\
P_{0}(u) U=\{x \in U: Q(v) x=x \text { for some tripotent } v \text { orthogonal to } u\} .
\end{gathered}
$$

In each case $k=2,1,0, P_{k}(u) U$ is determined by the quadratic products and orthogonality. Since $\phi(0)=0$, we have, by (2.1), and previous lemma

$$
\phi\left(P_{k}(u) U\right)=P_{k}(\phi(u)) V \quad \text { for } k=2,1,0 .
$$

Thus for any $x \in U$ there is $y \in V$ such that $\phi\left(P_{k}(u) x\right)=P_{k}(\phi(u)) y$, implying $P_{k}(\phi(u)) \phi\left(P_{k}(u) x\right)=P_{k}(\phi(u))^{2} y=\phi\left(P_{k}(u) x\right)$.

Corollary 2.5. If $\phi$ is as in Corollary 2.4, then the relations of collinearity and governing of tripotents are preserved by $\phi$.

Proof. Let $u$ and $v$ be tripotents of $U$. If $u \in P_{1}(v) U$ and $v \in P_{1}(u) U$, then, by Corollary 2.4, $\phi(u)$ and $\phi(v)$ are tripotents of $V$ and $\phi(u) \in P_{1}(\phi(v)) V$ and $\phi(v) \in P_{1}(\phi(u)) V$.

If $v \in P_{2}(u) U$ and $u \in P_{1}(v) U$, then, by Corollary $2.4 \phi(v) \in P_{2}(\phi(u)) V$ and $\phi(u) \in P_{1}(\phi(v)) V$.

Lemma 2.6. If $\phi$ is as in Lemma 2.3, then $\phi(-u)=-\phi(u)$ for arbitrary tripotent $u$ in $U$.

Proof. Set $v=\phi^{-1}((\phi(-u)+\phi(u)) / 2)$. Using the definition of $v$, the quadratic property of $\phi$ and $P_{2}(\phi(u))=P_{2}(\phi(-u)$, it is easy to verify that $Q(\phi(v)) \phi(v)$ $=\phi(v)$ and $\phi(v)=Q(\phi(v)) \phi(u)=\phi(Q(v) u)$. Thus

$$
\begin{aligned}
\phi(-v) & =\phi(-Q(v) u)=\phi(Q(v)(-u))=Q(\phi(v)) \phi(-u) \\
& =Q((\phi(-u)+\phi(u)) / 2) \phi(-u)=\phi(v)
\end{aligned}
$$

implying $-v=v$. Therefore $v=0$ and $\phi(-u)=-\phi(u)$.

Lemma 2.7. Let $U$ be a $J B W^{*}$-triple. Let $\phi$ is a bijective quadratic map from $U$ to another $J B^{*}$-triple $V$. Then for any two orthogonal tripotents $u$ and $v$ in $U$ and any $x$ and $y$ in $U$, we have

$$
\phi(Q(u) x+Q(v) y)=\phi(Q(u) x)+\phi(Q(v) y) .
$$

Proof. Let $z=Q(u) x+Q(v) y$. Observe that

$$
\begin{aligned}
\phi(z) & =\phi(Q(u+v) Q(u \pm v) z) \\
& =Q(\phi(u)+\phi(v)) Q(\phi(u) \pm \phi(v)) \phi(z) \\
& =Q(\phi(u)+\phi(v))[Q(\phi(u)) \phi(z)+Q(\phi(v)) \phi(z) \pm 2\{\phi(u) \phi(z) \phi(v)\}] .
\end{aligned}
$$


Since the identity holds for signs + and - , by averaging the two identities we will have

$$
\begin{aligned}
\phi(z) & =Q(\phi(u)+\phi(v))[Q(\phi(u))+Q(\phi(v))] \phi(z) \\
& =Q(\phi(u))^{2} \phi(z)+Q(\phi(v))^{2} \phi(z) \\
& =\phi\left(Q(u)^{2} z\right)+\phi\left(Q(v)^{2} z\right) \\
& =\phi(Q(u) x)+\phi(Q(v) y) .
\end{aligned}
$$

Corollary 2.8. Let $U, V$ and $\phi$ be as in Lemma 2.7 and let $x$ and $y$ be two orthogonal elements in $U$, i.e., $\{x y U\}=0$, then $\phi(x)$ is orthogonal to $\phi(y)$ and $\phi(x+y)=\phi(x)+\phi(y)$.

Proof. Let $u$ be the support tripotent of $x$. Then $x=Q(u) x, x=P_{2}(u) x$ and $y=P_{0}(u) y$. From Corollary 2.4 follows that $\phi(x)=P_{2}(\phi(u)) \phi(x)$ and $\phi(y)=$ $P_{0}(\phi(u)) \phi(y)$. Thus $\phi(x)$ is orthogonal to $\phi(y)$. Let $v$ be the support tripotent of $y$ then $y=Q(v) y$ and the result follows from the lemma.

Lemma 2.9. Let $U, V$ and $\phi$ be as in Lemma 2.7, and let $u$ and $w$ be tripotents in $U$ with u governed by $w$. Then for any $x$ and $y$ in $U$, we have

$$
\phi(Q(u) x+Q(u) y)=\phi(Q(u) x)+\phi(Q(u) y) .
$$

Proof. Let $v=Q(w) u, p=(u+v+w) / 2$ and $q=(u+v-w) / 2$. Then by Lemma 1.1, $p$ and $q$ are orthogonal tripotents. From (1.6), for any $z \in U$, we have

$$
Q(u) Q(p) Q(u)(4 z)=Q(Q(u) p)(4 z)=Q(u / 2)(4 z)=Q(u) z \text {. }
$$

Similarly, $Q(u) Q(q) Q(u)(4 z)=Q(u) z$. Thus by using Lemma 2.7 and (2.1), we have

$$
\begin{aligned}
\phi(Q(u) x+Q(u) y) & =\phi(Q(u)[Q(p) Q(u)(4 x)+Q(q) Q(u)(4 y)]) \\
& =Q(\phi(u)) \phi[Q(p) Q(u)(4 x)+Q(q) Q(u)(4 y)] \\
& =Q(\phi(u))[\phi(Q(p) Q(u)(4 x))+\phi(Q(q) Q(u)(4 y))] \\
& =\phi(Q(u) Q(p) Q(u)(4 x))+\phi(Q(u) Q(q) Q(u)(4 y)) \\
& =\phi(Q(u) x)+\phi(Q(u) y) .
\end{aligned}
$$

By use of Proposition 1.3, from this lemma, it is possible to prove additivity of $\phi$ in case when $U$ have no type $I_{1}$ summands. On the other hand any tripotent $u$ in a type $I_{1} J B W^{*}$-triple does not satisfy the conditions of previous lemma. Thus, we must treat such triples separately. With the help of the following lemma this will be done in Lemma 2.11 .

Lemma 2.10. Let $U$ and $V$ be $J B W^{*}$-triples and $U=\bigoplus_{\alpha \in A} U_{a}$ where $U_{a}$ is a $w^{*}$ - 
closed ideal of $U$. If $\phi$ is a bijective quadratic map from $U$ to $V$, then $V=$ $\bigoplus_{\alpha \in A} \phi\left(U_{\alpha}\right)$ where $\phi\left(U_{\alpha}\right)$ is also a $w^{*}$-closed ideal of $V$. Especially, if $x=\sum_{\alpha \in A} x_{\alpha}$ where $x_{\alpha} \in U_{\alpha}$ and $\phi(x)=\sum_{a \in A} \phi(x)_{\alpha}$ where $\phi(x)_{\alpha} \in \phi\left(U_{a}\right)$, then $\phi\left(x_{\alpha}\right)=\phi(x)_{\alpha}$ for every $\alpha \in A$.

Proof. By Corollary 2.8, we have $\phi\left(U_{\alpha}\right)^{\perp}=\phi\left(U_{\alpha}^{\perp}\right), \quad V=\phi\left(U_{\alpha}\right) \oplus \phi\left(U_{\alpha}\right)^{\perp}$ and $\phi\left(U_{\alpha}\right)=\left(\phi\left(U_{\alpha}\right)\right)^{\perp \perp}$. Thus $\phi\left(U_{\alpha}\right)$ is a summand in $V$ and a $w^{*}$-closed ideal of $V$. For any $x \in U$ let $u$ be the tripotent in the polar decomposition of $x$. Decompose $u$ into a sum of orthogonal tripotents $u_{\alpha} \in U_{\alpha}$. Then $x_{a}=P_{2}\left(u_{\alpha}\right) x, \phi(x)_{\alpha}$ $=P_{2}\left(\phi\left(u_{\alpha}\right)\right) \phi(x)$ and $\phi(x)_{\alpha}=\phi\left(x_{\alpha}\right)$ for $\alpha \in A$.

Lemma 2.11. Let $W$ be a JBW*-triple of type $I_{1}$ with no abelian summand and let $\phi$ be a bijective quadratic map from $W$ to a JBW*-triple $V$. Then $\phi$ is additive.

Proof. Since properties of being abelian and complete are preserved by $\phi$, $V=\phi(W)$ is of type $I_{1}$. Moreover from the classification of $J B W^{*}$-triples [10] $W$ can be decomposed into $W=\oplus W_{n}$ for some cardinal numbers $n$, where each $W_{n}$ is a $J B W^{*}$-triple of type $I_{1, n}$. By Lemma 2.10 it is enough to prove the proposition for $W_{n}$. Thus we will assume that $W$ is of type $I_{1, n}$. In this case there is a maximal collinear family of $n$ complete abelian tripotents $\left\{v_{j}\right\}_{j \in J}$ in $W$ such that $W=\overline{\operatorname{span}}\left\{W_{2}\left(v_{j}\right): j \in J\right\}$, or equivalently $\cap\left\{W_{1}\left(v_{j}\right): j \in J\right\}=0$.

We shall prove first that for any pair of collinear complete abelian tripotents $u$ and $v$ and any $x, y$ in $W$ we have

$$
\phi(Q(u) x+Q(v) y)=\phi(Q(u) x)+\phi(Q(v) y) .
$$

Denote $a=Q(u) x$ and $b=Q(v) y$. Complete, by use of $[10,2.7], u$ and $v$ to a maximal collinear family $\{u, v\} \cup\{u,\}_{j \in \tilde{J}}$ of complete abelian tripotents. Since the subspace span $\left\{W_{2}(u), W_{2}(v)\right\}=\bigcap_{j \in \tilde{J}} W_{1}\left(u_{j}\right)$ and $a+b$ belongs to this subspace, from Corollary 2.4 it follows that $\phi(a+b) \in \operatorname{span}\left\{V_{2}(\phi(u)), V_{2}(\phi(v))\{\right.$. Thus

$$
\phi(a+b)=\tilde{a}+\tilde{b},
$$

where $\tilde{a} \in V_{2}(\phi(u))$ and $\tilde{b} \in V_{2}(\phi(v))$. But since $a=Q(u)^{2}(a+b)$ and $b=Q(v)^{2}(a+b)$ we have

implying (2.3).

$$
\text { and } \begin{aligned}
& \phi(a)=\phi\left[Q(u)^{2}(a+b)\right]=Q(\phi(u))^{2} \phi(a+b)=\tilde{a} \\
& \phi(b)=\tilde{b},
\end{aligned}
$$

Note that $p=(u+v) / \sqrt{2}$ and $q=(u-v) / \sqrt{2}$ form a pair of collinear complete abelian tripotents. Moreover from (1.6), we have $Q(u)(x / 2)=Q(u / \sqrt{2}) x$ $=Q(Q(u) p) x=Q(u) Q(p) Q(u) x$ and $Q(u)(x / 2)=Q(u) Q(q) Q(u) x$. Thus for any $x$ and $y$ in $W$, using (2.3) for the collinear tripotents $p$ and $q$, we have 


$$
\begin{aligned}
\phi(Q(u) x+Q(u) y) & =\phi(Q(u)[Q(p) Q(u)(2 x)+Q(q) Q(u)(2 y)]) \\
& =Q(\phi(u)) \phi(Q(p) Q(u)(2 x)+Q(q) Q(u)(2 y)) \\
& =Q(\phi(u))[\phi(Q(p) Q(u)(2 x))+\phi(Q(q) Q(u)(2 y))] \\
& =\phi(Q(u) Q(p) Q(u)(2 x))+\phi(Q(u) Q(q) Q(u)(2 y)) \\
& =\phi(Q(u) x)+\phi(Q(u) y) .
\end{aligned}
$$

From this follows that $Q\left(v_{j}\right)(\phi(x)+\phi(y)-\phi(x+y))=0$ for all $j$ and thus $\phi(x)+$ $\phi(y)-\phi(x+y) \in \cap\left\{W_{1}\left(v_{\jmath}\right): j \in J\right\}=\{0\}$. So $\phi(x+y)=\phi(x)+\phi(y)$.

Now we are able to prove additivity of $\phi$ in the general case. Note that if $U=\mathbf{C}$ then $\phi(z)=z|z|$ is a quadratic map which is not additive. Therefore abelian direct summands must be excluded.

Theorem 2.12. Let $U$ be a JBW*-triple with no abelian direct summand, and let $\phi$ be a bijective quadratic map from $U$ to another $J B W^{*}$-triple $V$. Then $\phi$ is additive i.e.,

$$
\phi(x+y)=\phi(x)+\phi(y)
$$

for any $x$ and $y$ in $U$.

Proof. Decompose $U=U_{I_{1}}+\tilde{U}$ into a direct sum of a type $I_{1} J B W^{*}$-triple $U_{I_{1}}$ and a $J B W^{*}$-triple $\tilde{U}$ having no type $I_{1}$ summands. Then $\phi \mid U_{I_{1}}$ is a quadratic bijection from $U_{I_{1}}$ onto a type $I_{1}$ summands of $V$. Since $U_{I_{1}}$ has no abelian direct summand, Lemma 2.11 implies that $\phi \mid U_{I_{1}}$ is additive. Thus, using Lemma 2.10 it is enough to show that $\phi \mid \tilde{U}$ is additive.

Let $\tilde{x}=\phi(x)$ and $\tilde{y}=\phi(y)$ for $x, y \in \tilde{U}$. Then (2.7) is equivalent to $\phi^{-1}(\tilde{x})+$ $+\phi^{-1}(\tilde{y})=\phi^{-1}(\tilde{x}+\tilde{y})$. By Proposition 1.3 choose a complete family $\left\{f_{i}\right\}_{i \in I}$ in $\tilde{U}_{*}$ such that each $\left\{f_{i}\right\}$ has property $G$. Thus, to prove (2.7) it is enough to show that for each $i \in I$,

$$
\left\langle f_{i}, \phi^{-1}(\tilde{x}+\tilde{y})\right\rangle=\left\langle f_{i}, \phi^{-1}(\tilde{x})\right\rangle+\left\langle f_{i}, \phi^{-1}(\tilde{y})\right\rangle .
$$

Let $u=u\left(f_{i}\right)$. Then by (1.22), (2.8) will hold if $Q(u) \phi^{-1}(\tilde{x}+\tilde{y})=Q(u) \phi^{-1}(\tilde{x})+$ $Q(u) \phi^{-1}(\tilde{y})$ or

$$
\phi(Q(u) x+Q(u) y)=\phi(Q(u) x)+\phi(Q(u) y) .
$$

If $f_{i}$ had property $G$ then by Lemma 1.2, there exists a tripotent $w$ in $U$ such that $w$ governs $u$. Thus (2.9) follows from Lemma 2.9 and $\phi \mid \tilde{U}$ is additive.

Using the fact that $2\{x y z\}=Q(x+z) y-Q(x) y-Q(z) y$, from Theorem 2.12 if follows: 
Corollary 2.13. Let $\phi$ be as in Theorem 2.12. Then for any $x, y, z$ in $U$, we have

$$
\phi(\{x y z\})=\{\phi(x) \phi(y) \phi(z)\}
$$

Now we are able to show that $\phi$ is real homogeneous.

Proposition 2.14. Let $U$ and $V$ be $J B W^{*}$-triples and suppose $U$ has no abelian direct summand. Let $\phi$ be a bijective quadratic map from $U$ to $V$. Then $\phi$ is a real linear isometry i.e.,

$$
\begin{gathered}
\phi(\lambda x)=\lambda \phi(x) \quad \text { for } \quad x \in U, \lambda \in \mathbf{R}, \\
\|\phi(x)\|=\|x\| \quad \text { for } x \in U .
\end{gathered}
$$

Proof. Let $u$ be any tripotent of $U$. Then $P_{2}(u) U$ is a $J B W^{*}$-algebra with unit $u$ under the operations $x \circ y:=\{x u y\}$ and adjoint $\#: y \rightarrow y^{\#}:=\{u y u\}$ for $x$ and $y$ in $P_{2}(u) U$.

Since any positive element in $P_{2}(u) U$ is of the form $y \circ y=\{y u y\}$ for self adjoint $y$ (i.e., $y=\{u y u\}$ ), $\phi$ maps positive elements of $P_{2}(u) U$ into positive elements of $P_{2}(\phi(u)) V$. Moreover, if $a$ and $b$ are in $P_{2}(u) U$ and $a \leqq b$ in the sense of $J B W^{*}$-algebra, then $\phi(a) \leqq \phi(b)$ in $P_{2}(\phi(u)) V$. From the additivity of $\phi$, it follows that (2.10) holds for all positive rational $\lambda$. Since $\phi$ is additive and order preserving in the sense of $J B W^{*}$-algebra, $0 \leqq \phi(\lambda u) \leqq \phi\left([1 / \lambda]^{-1} u\right)=[1 / \lambda]^{-1} \phi(u)$ holds for $0<\lambda<1$ where $[1 / \lambda]$ denotes the integral part of $1 / \lambda$. Combining with the condition $\phi(-\lambda u)=-\phi(\lambda u)$ the map $\lambda \rightarrow \phi(\lambda u)$ is continuous at $\lambda=0$, hence also continuous on $\mathbf{R}$. Thus we have

$$
\phi(\lambda u)=\lambda \phi(u) \quad \text { for any } \lambda \in \mathbf{R} .
$$

Finally, for any $x \in U$, let $u$ be the support tripotent of $x$. Then for any $\lambda>0$, with $\mu=\sqrt{\lambda}$ we have

$$
\begin{aligned}
\phi(\lambda x) & =\phi(\lambda\{u x u\})=\phi(\{(\mu u) x(\mu u)\}) \\
& =\{\phi(\mu u) \phi(x) \phi(\mu u)\}=\mu^{2}\{\phi(u) \phi(x) \phi(u)\} \\
& =\lambda \phi(\{u x u\})=\lambda \phi(x) .
\end{aligned}
$$

Since $\phi$ is bijective, real linear and positive from the $J B W^{*}$-algebra $P_{2}(u) U$ to the $J B W^{*}$-algebra $P_{2}(\phi(u)) V$, and by [9: Proposition 3.3.10] $\|x\|=\inf \{\lambda>0:-\lambda u$ $\leqq x \leqq \lambda u\}$ for $x=\{u x u\} \in P_{2}(u) U, \phi$ is norm preserving.

A bijective quadratic map $\phi$ need not be complex linear in general but the following theorem shows that such $\phi$ is an orthogonal sum of linear and conjugate linear maps.

Theorem 2.15. Let $U$ be a JBW*-triple with no abelian direct summand, let 
$\phi$ be a bijective quadratic map from $U$ to another $J B W^{*}$-triple, let $W_{1}=\{x \in U$ : $\phi(\xi x)=\xi \phi(x)$ for all $\xi \in \mathbf{C}\}$ and let $W_{2}=\{x \in U: \phi(\xi x)=\bar{\xi} \phi(x)$ for all $\xi \in \mathbf{C}\}$. Then $W_{i}(i=1,2)$ are orthogonal $w^{*}$-closed ideals in $U$ such that $U=W_{1} \oplus W_{2}$ and $\phi=$ $\phi_{1}+\phi_{2}$ where

$$
\phi_{\imath}(x)= \begin{cases}\phi(x) & x \in W_{i} \\ 0 & x \in W_{j}(j \neq i) .\end{cases}
$$

Proof. For any $x$ and $y$ in $U$, for any $a \in W_{1}$, and for any $\xi \in \mathbf{C}$, by Corollary 2.13 ,

$$
\begin{aligned}
\phi(\xi\{x y a\}) & =\phi(\{x y(\xi a)\})=\{\phi(x) \phi(y) \phi(\xi a)\} \\
& =\xi\{\phi(x) \phi(y) \phi(a)\}=\xi \phi(\{x y a\})
\end{aligned}
$$

and similarly,

$$
\phi(\xi\{x a y\})=\xi \phi(\{x a y\}) .
$$

Thus $W_{1}$ is an ideal of $U$. Similarly, $W_{2}$ is also an ideal of $U$ and $W_{1} \cap W_{2}=\{0\}$ implies that $W_{1}$ is orthogonal to $W_{2}$.

Let $a \in\left(W_{1}\right)^{\perp}$. Then the support tripotent $u=u(a)$ of $a$ belongs to $\left(W_{1}\right)^{\perp}$. Since $W_{2}$ is an ideal, to show that $a \in W_{2}$ it is enough to prove that $u \in W_{2}$ or $\phi(i u)+i \phi(u)=0$. Define $w=\phi^{-1}((\phi(i u)+i \phi(u)) /(2 i))$. It is easy to check that $\phi(w)$ and $\phi(u)-\phi(w)$ are orthogonal tripotents. Thus

$$
\begin{aligned}
\phi(i w) & =\phi\left(i P_{2}(w) u\right)=\phi\left(P_{2}(w)(i u)\right) \\
& =P_{2}(\phi(w)) \phi(i u) \\
& =P_{2}(\phi(w))[i \phi(w)-i(\phi(u)-\phi(w))] \\
& =i P_{2}(\phi(w)) \phi(w)=i \phi(w) .
\end{aligned}
$$

But by Corollary $2.8 \phi(w) \in \phi\left(W_{1}^{\perp}\right) \subset \phi\left(W_{1}\right)^{\perp}$ implying $\phi(w)=0$ or $\phi(i u)=-i \phi(u)$. Thus $u \in W_{2}$ and $a=\{u a u\} \in W_{2}$. Therefore $\left(W_{1}\right)^{\perp} \subset W_{2}$ or $W_{2}=\left(W_{1}\right)^{\perp}$ implies $U=W_{1} \oplus W_{2}$ and $W_{i}(i=1,2)$ are $w^{*}$-closed ideals.

\section{$\S 3$. Applications}

As an application to von Neumann algebras, we have the following theorem, which is a generalization of a result of the second named author [7].

Theorem 3.1. Let $M$ and $N$ be von Neumann algebras and suppose $M$ has no abelian direct summand. Let $\phi$ be a bijective quadratic map (i.e., $\phi\left(a b^{*} a\right)=$ $\left.\phi(a) \phi(b)^{*} \phi(a)\right)$ without assuming additivity of $\phi$ from $M$ to $N$. Then $\phi$ is a real linear isometry. Moreover, there exist four central projections $p_{1}, p_{2}, p_{3}, p_{4}$ in $M$ such that $p_{1}+p_{2}+p_{3}+p_{4}=1$ and 
$\phi$ is a linear *-ring isomorphism on $M p_{1}$;

$\phi$ is a linear *-ring anti-isomorphism on $M p_{2}$;

$\phi$ is a conjugate linear $*_{-r i n g}$ isomorphism on $\mathrm{Mp}_{3}$;

$\phi$ is a conjugate linear $*_{-}$ring anti-isomorphism on $M p_{4}$.

Proof. Under the triple product $\{a b c\}:=\left(a b^{*} c+c b^{*} a\right) / 2, M$ is a $J B W^{*}$-triple. By Proposition 2.14 and Theorem 2.15, there exist two $w^{*}$-closed orthogonal ideals $M_{1}$ and $M_{2}$ in triple sense such that $M=M_{1} \oplus M_{2}, \phi \mid M_{1}$ is linear and $\phi \mid M_{2}$ is conjugate linear. Obviously, $M_{i}$ are von Neumann subalgebras of $M$ with central projections $e_{i}$ as an identity $(i=1,2)$.

From the definition of $\phi$ we have $\phi\left(b^{*}\right)=\phi(b)^{*}$ for any $b \in M$, i. e., $\phi$ is * preserving. Define $\phi(x)=\phi\left(x e_{1}\right)+\phi\left(x e_{2}\right)^{*}$. Then $\phi$ is a $C^{*}$-isomorphism between $M$ and $N$. Hence there exist central projections $f_{1}$ and $f_{2}$ such that $f_{1}+f_{2}=1$, $\phi \mid M f_{1}$ is *-ring isomorphism and $\psi \mid M f_{2}$ is *-ring anti-isomorphism [12]. Therefore if we put $p_{1}=e_{1} f_{1}, p_{2}=e_{1} f_{2}, p_{3}=e_{2} f_{2}$ and $p_{4}=e_{2} f_{1}$, then $p_{1}, p_{2}, p_{3}$ and $p_{4}$ satisfy all the requirements of the theorem.

The following Corollary is a special case of [7].

Corollary 3.2. Let $M$ and $N$ be von Neumann algebras and suppose $M$ has no abelian summand. Let $\phi$ be an *-semigroup isomorphism from $M$ to $N$, that is, $\phi$ is a bijective map from $M$ to $N$ which satisfies $\phi(a b)=\phi(a) \dot{\phi}(b)$ and $\phi\left(a^{*}\right)=$ $\phi(a)^{*}$. Then $\phi$ is an orthogonal sum of a linear and a conjugate linear *-isomorphism.

Proof. Obviously, a *-semigroup isomorphism $\phi$ is a bijective quadratic map from $M$ to $N$. Applying Theorem 3.1, we have the conclusion.

We finish this paper with the problem discussed in the introduction:

Proposition 3.3. Let $G$ be a quadratic semigroup (defined by Definition 2) and $\pi_{1}, \pi_{2}$ be two faithful representations of $G$ onto $J B W^{*}$-triples with no abelian direct summand $U_{1}$ and $U_{2}$ respectively, then the induced map $\pi_{2} \circ \pi_{1}^{-1}$ between this two representations is a real linear isometry.

Proof. Obviously $\pi_{2}^{\circ} \pi_{1}^{-1}$ satisfies the conditions of Proposition 2.14 and thus the conclusion follows.

\section{References}

[1] Barton, T. and Timoney, R., Weak*-continuity of Jordan triple products and its applications, Math. Scand., 59 (1986), 177-191.

[2] Dineen, S., Complete holomorphic vector fields on the second dual of a Banach space, Math. Scand., 59 (1986), 131-192. 
[3] Dang, T. and Friedman, Y., Classification of $J B W^{*}$-triple factors and applications, Math. Scand., 61 (1987), 292-330.

[4] Emch, G. G., Algebraic methods in statistical mechanics and quantum field theory, Wiley-Interscience, New York, 1972.

[5] Friedman, Y. and Russo, B., Structure of the predual of a $J B W^{*}$-triple, Journal fur die reine und angewandte math., 356 (1985), 67-89.

[6] Gunnaydin, M., Quadratic Jordan formulation of Quantum Mechanics. Construction of Lie (super) algebras from Jordan (super) algebras, Viii Intern. Colloq. on Group Theoretical Methods in Physics, Kiriat Anavim, Israel, Ann. Israel Phys. Soc., 3 (1980), 279-296.

[7] Hakeda, J., Additivity of * semigroup isomorphisms among *-algebra, Bull. London Math. Soc., 18 (1986), 51-56.

[8] Hakeda, J. and Saito, K., Additivity of Jordan * maps between operator algebras, J. Math. Soc. Japan, 38 (1986), 403-408.

[9] Hanche-Olsen, H. and Størmer, E., Jordan Operator Algebras, Pitman, London, 1984.

[10] Horn, G., Classification of $J B W^{*}$-triples of type I, Math. Z., 196 (1987), 271-291.

[11] —, Klassifikation der $J B W^{*}$-triple vom Type $I$, Dissertation. Tübingen, 1984.

[12] - Characterization of the predual and ideal structure of a $J B W^{*}$-triple, Math. Scand. (to appear).

[13] Jordan, P., von Neumann, J. and Wigner, E., On an algebraic generalization of the quantum mechanical formalism, Annals Math., 35 (1934), 29-64.

[14] Kadison, R. V., Isometries of operator algebras, Ann. Math., (2) 59 (1951), 325-338.

[15] Kaup, W., A Riemann mapping theorem for bounded symmetric domain in complex Banach space, Math. Z., 183 (1983), 503-529.

[16] Loos, O., Bounded symmetric domains and Jordan pairs, Lecture Notes, University of California, Irvine, 1977.

[17] - Jordan pairs, Lecture notes in Math., 460, Springer-Verlag, 1975.

[18] McCrimmon, K., Compatible Peirce decompositions of Jordan triple system, Pacific Jour. Math., 103 (1982), 57-102.

[19] Segal, I.E., Postulates for general quantum mechanics, Annals of Math., 48 (1947), No. 4 , 930-948.

[20] Upmeier, H., Symmetric Banach manifolds and Jordan $C^{*}$-algebras, North-Holland, 1985. 